\title{
Uma abordagem sobre os recursos e incentivos para inovação
}

\author{
An approach on resources and incentives for innovation \\ Un enfoque sobre los recursos e incentivos para la innovación
}

Edson Lauro Marchini

ORCID: https://orcid.org/0000-0001-9996-9150 Universidade Estadual de Maringá, Brasil

E-mail: edson.marchini@gmail.com

José Roberto Dias Pereira

ORCID: https://orcid.org/0000-0001-8281-2376 Universidade Estadual de Maringá, Brasil E-mail: jrdp@dfi.uem.br

\begin{abstract}
Resumo
Objetivo: Este trabalho busca através de uma pesquisa quantitativa descritiva, apresentar um comparativo entre empresas que inovaram e empresas que utilizaram recursos financeiros disponíveis para tal e justificar a divergência entre os indicadores. Métodos: Esta pesquisa quantitativa descritiva realizou o levantamento de dados secundários disponibilizados pelo IBGE nos resultados PINTEC entre 2014 e 2017 e dos dados de desembolso do BNDES para o mesmo intervalo temporal. De posse dos dados, filtros foram aplicados para segmentar dados de operações com créditos classificados como inovação, em seguida calculou-se o percentual para compreender o grau de utilização dos recursos disponíveis para inovação. Resultados: Os resultados encontrados indicaram que 33,6\% das empresas pesquisadas foram inovadoras em produtos ou processos, dados que indicam uma redução em relação aos dois triênios anteriores. Dentre essas empresas, a maioria (89\%) utilizou recursos próprios para o desenvolvimento das atividades. Conclusão: Pode-se constatar uma diferença nos apontamentos dos tipos de apoios declarados pelos indicadores e as dificuldades apontadas pelas empresas para a implementação de inovação.
\end{abstract}

Palavras-chave: Recursos para inovação; Incentivos para inovação; Desembolso com inovação.

\begin{abstract}
Aim: This paper seeks, through a descriptive quantitative research, to present a comparison between companies that innovated and companies that used financial resources available for this purpose and justify the divergence between the indicators. Methods: This descriptive quantitative research carried out a survey of secondary data made available by IBGE on PINTEC results between 2014 and 2017 and BNDES disbursement data for the same time interval. In possession of the data, filters were applied to segment data of operations with credits classified as innovation, then the percentage was calculated to understand the degree of use of available resources for innovation. Results: The results found indicated that $33.6 \%$ of the surveyed companies were innovative in products or processes, data that indicates a reduction in relation to the previous two trienniums. Among these companies, most (89\%) used their own resources for the development of activities. Conclusion: We can see a difference in the types of support declared by the indicators and the difficulties pointed out by companies for the implementation of innovation.
\end{abstract}

Keywords: Resources for innovation; Incentives for innovation; Innovation disbursements.

\section{Resumen}

Objetivo: Este trabajo pretende, a través de una investigación cuantitativa descriptiva, presentar una comparación entre las empresas que innovaron y las que utilizaron los recursos financieros disponibles para ello y justificar la divergencia entre los indicadores. Métodos: Esta investigación cuantitativa descriptiva realizó un estudio de datos secundarios puestos a disposición por el IBGE sobre los resultados del PINTEC entre 2014 y 2017 y los datos de desembolso del BNDES para el mismo intervalo de tiempo. En posesión de los datos, se aplicaron filtros para segmentar los datos de las operaciones con créditos clasificados como innovación, y luego se calculó el porcentaje para conocer el grado de utilización de los recursos disponibles para la innovación. Resultados: Los resultados encontrados indican que el 33,6\% de las empresas encuestadas son innovadoras en productos o procesos, dato que indica una reducción en relación a los dos trienios anteriores. Entre estas empresas, la mayoría (89\%) utilizó recursos propios para el desarrollo de las actividades. Conclusión: Se observa una diferencia en las indicaciones de los tipos de apoyo declarados por los indicadores y las dificultades señaladas por las empresas para la implementación de la innovación. Palabras clave: Recursos para la innovación; Incentivos para la innovación; Desembolsos para la innovación. 


\section{Introdução}

A necessidade de se destacar na competitividade econômica mundial está intimamente ligada à capacidade da nação em inovar e essa habilidade, por sua vez, resulta dos esforços e o investimento em pesquisa e desenvolvimento - p\&d (Calmanovici, 2011).

A difusão da inovação é um elemento determinante para estimular o desenvolvimento econômico e, consequentemente, proporcionar o avanço técnico em processo evolucionista (Schumpeter, 1982). Lall (1992) destaca que o processo de inovar depende de uma capacidade tecnologia, (Fagerberg \& Srholec, 2008, apud Porto \& Memória, 2019) conceitua que a capacidade tecnológica faz referência na capacidade de desenvolver e explorar o conhecimento comercialmente.

A política industrial brasileira que antecede na década de 80 se baseava na criação da capacidade física, para atender o mercado interno e substituir as importações. O Estado criando empresas, atraindo investimentos externos, com garantias de uma proteção contra a competição internacional, oferecia um grande mercado interno como garantia. Estatais, protecionismo, incentivos fiscais, subsídios e creditícios marcaram a década. Nos anos seguintes, o governo promoveu a abertura para o mercado externo reduzindo a proteção industrial brasileira e expondo o mercado interno a concorrência internacional (Daher \& Salerno, 2006).

O Brasil redirecionou seus passos na evolução para inovação e o reconhecimento da importância da inovação resultou em mudanças na forma de atuação das empresas, instituições de ensino, de pesquisa e organizações do setor público (Veloso \& Nogueira, 2006).

O papel do Estado, segundo Guimarães (2000), é implementar uma política de Ciência e Tecnologia (C\&T) compatível com a meta estratégica de criar um sistema nacional de inovações, medidas e instrumentos que satisfaçam suas demandas e superar os obstáculos que se colocam em seu caminho.

Em 1991, em substituição à reserva de mercado, o Brasil implementou uma política de informática (Lei n8.248/91) buscando incentivar o comprometimento das empresas com produções locais e desenvolvimento de atividades de pesquisa, desenvolvimento e inovação (pd\&i), através de incentivo fiscal por meio da isenção do imposto sobre produtos industrializados (IPI) (Kannebley Jr \& Porto, 2012).

Matias-Pereira (2015) destaca que o país deve esforçar-se para alcançar a soberania tecnológica, e isso requer passar a otimizar ao máximo a ciência, a tecnologia e a inovação tecnológica, buscar o desenvolvimento sustentável e criar condições para atender as demandas crescentes, o que requer implementações de políticas de Ciências, Tecnologia e Inovação (CT\&I) consistentes e duradouras.

O Brasil segue desenvolvendo seu papel de incentivo ao crescimento tecnológico criando instrumentos legais como a Lei de Inovação Tecnológica (Lei. N¹0.973/04), que tem sua organização formatada em torno de 3 eixos: i) a constituição de ambiente adequado estratégicos para a parceria entre universidades, Instituição de Ciência e Tecnologia (ICT) e empresas; ii) o estímulo à participação de ICT no processo de inovação e; iii) o incentivo à inovação na empresa através de subvenção econômica para o desenvolvimento tecnológico e através do uso do poder de compra governamental (Porto \& Memória, 2019).

Em 2016, foi instaurada a Lei $N^{\circ} 13.243$, que promove alterações na Lei $N^{\circ} 10.973 / 04$, dentre outras, proporcionando adequações à Lei de Inovação Tecnológica, expandindo o conceito de ICT a entidades privadas sem fins lucrativos, sendo considerada o novo marco Legal da Inovação (Rauen, 2017).

A Lei do Bem, (Lei. 11.196/05), por sua vez, permite a dedução direta dos dispêndios em pesquisa e desenvolvimento (PD\&I) do lucro das firmas, alterando o custo de uso do capital orientado a esse investimento. O objetivo principal destes benefícios fiscais previstos na Lei do Bem não é a inovação, mas as atividades de pesquisa e desenvolvimento de inovação tecnológica (PD\&I), ou seja, atividades que buscam a aquisição de novos conhecimentos e sobre as quais incidem os riscos tecnológicos (ANPEI, 2017). 
Como toda a atividade de P\&D é passível de riscos tecnológicos, o Estado pode compartilhar esses riscos com os empresários, porém a empresa deverá comprovar sua capacidade de investir em P\&D (Porto \& Memória, 2019). Calmanovici (2011) justifica que a baixa adesão das empresas aos principais instrumentos de apoio às atividades de P\&D pode ser explicada, em muitos casos, pela restrição ao uso dos benefícios fiscais (Lei do Bem) às empresas de lucro real, e isso representa apenas $8 \%$ de empresas que se encaixam neste regime. As demais $92 \%$ das empresas são optantes pelo regime de lucro presumido, representando a grande maioria das micro e pequenas empresas de base tecnológicas.

Scaff e Pereira (2021) destacam que a conformidade legal e operação inovativa são entraves para que as empresas de pequeno porte consigam se adequar as exigências legais para inovar constantemente para a sobrevivência em uma economia de mercado. Migrar para o regime de lucro real é complexo e oneroso, suportar o custo da operação inovativa sem benefícios fiscais ou simplesmente optar por não inovar, acabam se tornando as vias recorridas para as empresas de pequeno porte.

Segundo Ribeiro (2006), o Brasil tem a contradição de apresentar características tecnológicas típicas de países em desenvolvimento, com a capacidade para desenvolver e utilizar tecnologias avançadas em várias áreas do conhecimento.

A Lei de Inovação permite à administração pública financiar e subsidiar, em casos específicos, diretamente a inovação nas empresas. Estas, por sua vez, podem disputar os recursos de agências de fomento para seus projetos tecnológicos, que normalmente possuem uma parcela subsidiada, com recursos não reembolsáveis pelo Estado. O BNDES, por exemplo, passou a oferecer linhas especiais de financiamento à atividade inovadora para empresas brasileiras. Nos Estados, fundações de apoio à pesquisa também ofertam crédito, em geral mais focado na atividade científica e na relação universidade/empresa, além da Financiadora de Estudos e Projetos, do Ministério da Ciência e Tecnologia (Finep/MCT) (IPEA,2008).

Um fator determinante para o entendimento dos recursos utilizados é o levantamento dos dados de inovação e a compreensão melhor dessas atividades e sua relação com o crescimento econômico (De Oslo, 2005).

Diante de todo o esforço dedicado, o governo assume a necessidade de informações estatísticas mais relevantes que possibilitam tomadas de decisões para elaboração de políticas mais eficazes, garantindo o crescimento da produtividade e aumento das oportunidades no concorrido mercado mundial. Assim como em outros países, o Brasil cria seus instrumentos para mensurar as atividades de inovação tecnológica desenvolvidas pelas empresas sediadas no país e, através do Instituto Brasileiro de Geografia e Estatística (IBGE), com o apoio da Financiadora de Estudos e Projetos (FINEP) e do Ministério da Ciência e Tecnologia (MCT), desde de 2000, tem realizado a Pesquisa Industrial de Inovação (PINTEC), objetivando quantificar as atividades de inovação e seus resultados nas empresas industriais (Vicente \& Lopes, 2015).

Diante dos fatos expostos, tanto para o incentivo ao desenvolvimento da inovação assim como para os resultados levantados pela pesquisa do IBGE, questiona-se a relação dos indicadores das empresas que inovaram, segundo a PINTEC, comparado com as que utilizaram os recursos financeiros do BNDES destinados à inovação, e o número reduzido de empresas que utilizam os recursos financeiros disponíveis para a projetos inovadores. Desta forma este trabalho tem como objetivo explicar as relações entre os desembolsos para inovação e empresas que inovaram e, entender o grau de utilização dos recursos disponíveis para inovação.

\section{Procedimentos Metodológicos}

Este trabalho é classificado como uma pesquisa quantitativa descritiva, através do levantamento de dados secundários disponíveis pelo IBGE nos resultados da PINTEC 2014 e 2017, e dos dados de desembolso do BNDES para o mesmo intervalo temporal.

No método quantitativo, são coletados os dados numéricos ou quantitativos através de medições de grandezas e, por meio da metrologia obtém-se números com suas respectivas unidades. Por meio de técnicas matemáticas como o caso de 
porcentagens, estatísticas e probabilidades, o conjunto de dados são analisados possibilitando a previsão dos acontecimentos (Pereira et al, 2018).

As pesquisas descritivas têm como objetivo primordial a descrição das características de determinada população ou fenômeno ou, então, o estabelecimento de relações entre variáveis. Também são pesquisas descritivas aquelas que visam descobrir a existência de associações entre variáveis (Gil, 2002).

Foram realizadas buscas de informações nas instituições governamentais que apresentam dados de usuários de projetos de inovação: FIEP, BNDES e IBGE, sendo este as pesquisas PINTEC 2014 e 2017. Nas planilhas de dados de desempenho de operações do BNDES foram coletados os dados pesquisados, aplicados filtros para segmentar os dados de operação com créditos classificados como inovação e calculado o percentual.

\section{Resultados e Discussões}

A pesquisa da PINTEC 2017, que cobriu o triênio 2015-2017, apresentando um comparativo com os dois triênios anteriores, indicou que 33,6\% das empresas pesquisadas foram inovadoras em produto ou processo. Esse número representa uma redução de 2,4 pontos percentuais (p.p) em relação ao triênio anterior e 2,1 p.p em relação ao triênio 2009-2011, sugerindo um período de maior recrudescimento das dificuldades enfrentadas pelas empresas para a realização de inovação (IBGE, 2020a).

De acordo como o informativo PINTEC 2017, as empresas inovadoras utilizaram desembolsos na ordem de R \$ 67,3 bilhões em 2017, representando 1,95\% da receita líquida, onde $\mathrm{R} \$ 25,6$ bilhões foram investidos em atividades internas de P\&D, R \$ 21,2 bilhões em aquisição de máquinas e equipamentos e R \$ 7,0 bilhões em atividades externas de P\&D. (IBGE,2020a).

De acordo com os resultados em desembolso apresentado pelo BNDES, para o mesmo triênio somam-se um total de R\$ 11,9 bilhões destinados à inovação, conforme apontado na tabela 1, o qual representa 17,7\% do valor apontado pelo IBGE.

Segundo o mesmo relatório do IBGE (2020b), no triênio 2015-2017 apenas 4,7\% das empresas inovadoras utilizaram incentivos da Lei de P\&D e Inovação Tecnológica, apesar de nesse caso ter havido um crescimento de 1,2 p.p em relação ao triênio anterior e 2,0 p.p em relação à 2009-2011.

Com relação ao benefício exclusivo da Lei do Bem (Lei n.11.196/2005) houve uma evolução de 3,2\% (2012-2014) para 4,3\% (2015-2017) de empresas que usufruíram do benefício (IBGE, 2020a).

Para o triênio anterior, 2012-2014, houve investimento total de R \$ 81,5 bilhões realizados por empresas com atividades inovadoras, sendo 30,3\% com gastos em atividades internas de P\&D, segundo a PINTEC-2014 (IBGE, 2016).

De acordo como os dados informados pelo BNDES referente aos recursos utilizados pelas empresas nos anos de 2012 a 2017, são apontados os valores direcionados à utilização com projetos classificados como inovação, demonstrados na tabela 1 .

Tabela 1: Recursos utilizados por empresas entre 2012 a 2017.

\begin{tabular}{cccc}
\hline Ano & Total milhões & Inovação total milhões & Recursos para inovação \\
& $(\mathrm{R} \$)$ & $(\mathrm{R} \$)$ & $3,206 \%$ \\
\hline 2017 & $70.750,79$ & $2.268,55$ & $4,106 \%$ \\
2016 & $88.256,50$ & $3.624,17$ & $4,429 \%$ \\
2015 & $135.942,05$ & $6.020,22$ & $3,165 \%$ \\
2014 & $187.836,87$ & $5.945,73$ & $2,739 \%$ \\
2013 & $190.419,04$ & $5.214,91$ & $2,108 \%$ \\
2012 & $155.992,27$ & $3.288,41$ & 0 \\
\hline
\end{tabular}

Fonte: Elaborada pelo autor a partir de BNDES (2020). 
Confrontando os dados coletados de números de empresas que utilizaram recursos do BNDES para inovação com os dados do PINTEC para o mesmo período, observa-se uma desproporcionalidade no percentual, apresentada na tabela 2.

Tabela 2: Empresas que inovaram segundo BNDES e IBGE.

\begin{tabular}{|c|c|c|c|c|c|c|}
\hline \multicolumn{3}{|c|}{ BNDES } & \multicolumn{4}{|c|}{ IBGE } \\
\hline Ano & Total de empresas & $\begin{array}{l}\text { Total de } \\
\text { empresas que } \\
\text { utilizaram } \\
\text { recursos com } \\
\text { inovação }\end{array}$ & $\begin{array}{c}(\%) \text { de empresas } \\
\text { com recursos com } \\
\text { inovação }\end{array}$ & $\begin{array}{c}\text { Total de empresas } \\
\text { PINTEC }\end{array}$ & $\begin{array}{c}\text { Total de } \\
\text { empresas que } \\
\text { inovaram - } \\
\text { PINTEC }\end{array}$ & $(\%)$ que inovaram \\
\hline 2017 & 155616 & 1533 & $0,985 \%$ & & & \\
\hline 2016 & 199073 & 1837 & $0,923 \%$ & 116.962 & 39.329 & $33,625 \%$ \\
\hline 2015 & 277068 & 1369 & $0,494 \%$ & & & \\
\hline 2014 & 327779 & 930 & $0,284 \%$ & & & \\
\hline 2013 & 340581 & 488 & $0,143 \%$ & 132.529 & 47.693 & $35,987 \%$ \\
\hline 2012 & 304789 & 270 & $0,089 \%$ & & & \\
\hline
\end{tabular}

Nota: PINTEC - Pesquisa de Inovação Tecnológica. Fonte: Elaborada pelo autor a partir de BNDES, (2020) e IBGE (2020b, 2020c)

No quesito P\&D, para o triênio 2015-2017, tanto em atividades internas quanto externas, 89\% das empresas utilizaram recursos próprios para desenvolvimento de suas atividades (IBGE, 2020c).

A captação de recursos externos de acordo com os dados explorados pela FIEP para o ano de 2019 foi de $33 \%$ em um universo de 906 empresas pesquisadas, sendo que as 67\% restantes informaram terem utilizados recursos próprios.

O detalhamento dos tipos de fontes externas utilizadas são apresentados no Gráfico 1.

Gráfico 1: Tipo de fontes externas utilizadas na captação de recursos.

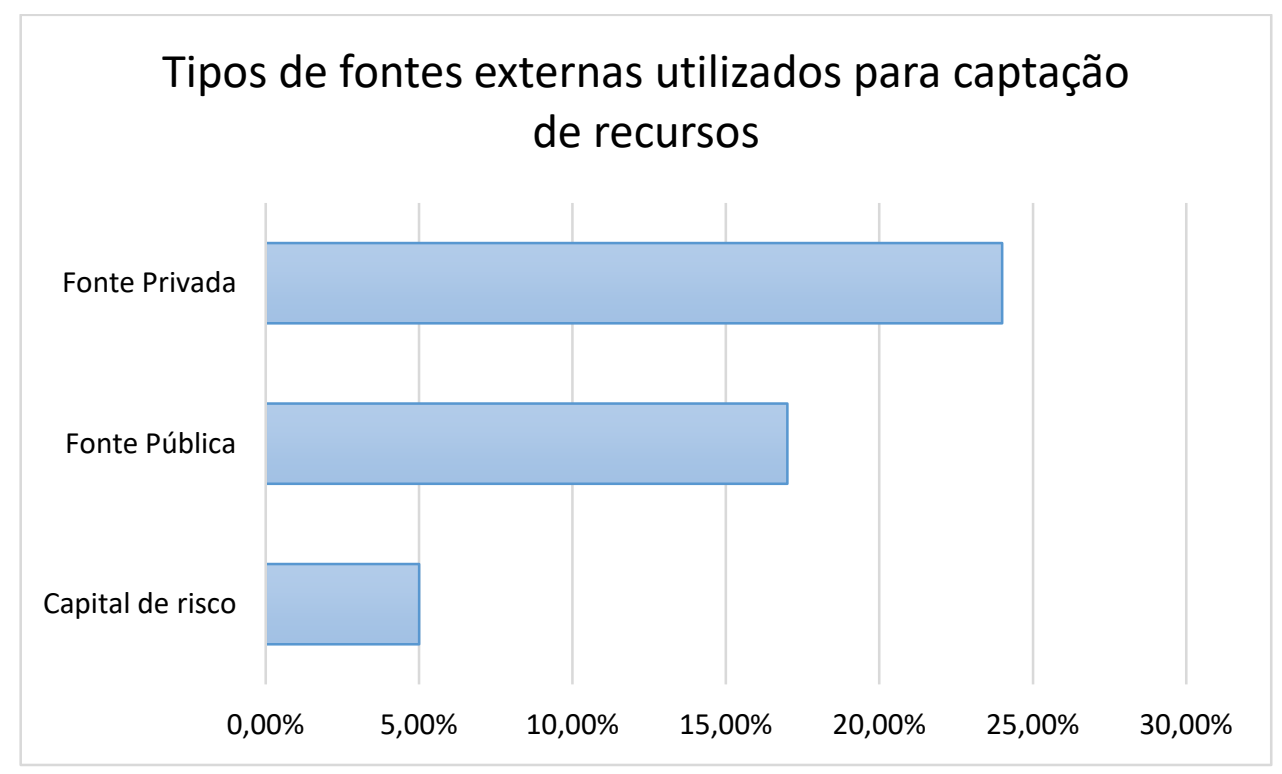

Fonte: Elaborado pelo autor conforme dados FIEP (2019).

Referente aos dados do IBGE, são apresentados no gráfico 2 o número de empresas que implementaram inovação com apoio do governo, segmentados por tipo de apoio, referentes às pesquisas PINTEC 2014 e 2017. 
Gráfico 2: Empresas que implementaram inovações com apoio do governo, por tipo de apoio.

\section{Número de empresas que implementaram inovações com apoio do governo, por tipo de apoio.}

Subvenção econômica

Outros

Incentivo fiscal - a Pesquisa e

Desenvolvimento

Incentivo fiscal - Lei da Informática

Financiamento - a projetos de Pesquisa e Desenvolvimento e inovação...

Financiamento - a projetos de Pesquisa e Desenvolvimento e inovação...

Financiamento - a compra de máquinas e equipamentos utilizados para inovar

Compras públicas

Bolsas oferecidas pelas fundações de amparo à pesquisa e RHAE/CNPq...

Aporte de capital de risco

$\square$ Pintec $2014 \quad \square$ Pintec 2017
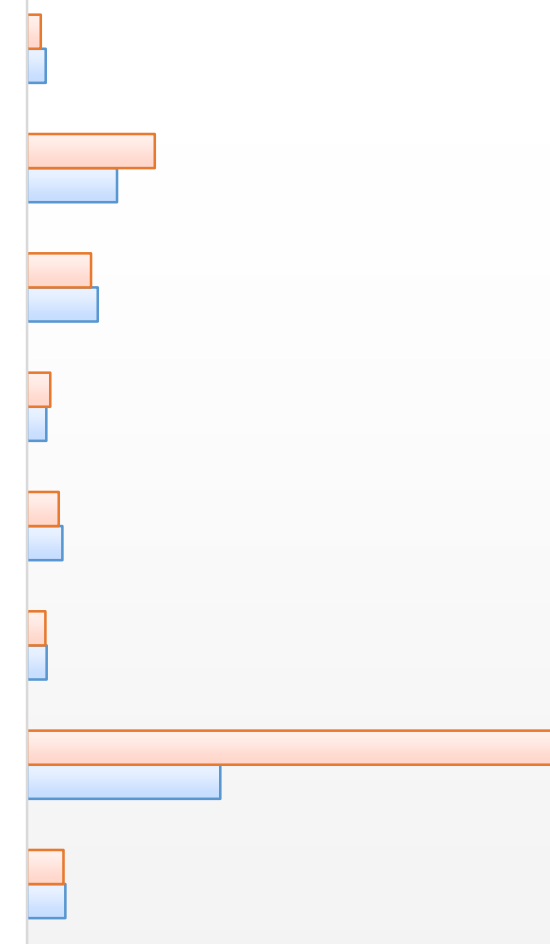

Nota: RHAE - Recursos Humanos em Áreas Estratégicas, CNPq - Conselho Nacional de Desenvolvimento Científico e Tecnológico.

Fonte: Elaborado pelo autor a partir de - IBGE (2020b, 2020c)

Nos dados coletados pela pesquisa PINTEC, os financiamentos de máquinas e equipamentos estão relacionados como investimento para inovação, enquanto que nos dados do BNDES esse desembolso é destacado no produto Finame e em sua maioria não são contabilizados como inovação.

Para o triênio 2015-2017 na pesquisa PINTEC, foram totalizadas 5086 empresas que adquiriam máquinas e equipamentos para fins de inovação, enquanto que no BNDES para o mesmo período foram contabilizadas apenas 287 empresas com o mesmo objetivo.

Essa classificação sugere que a liberação dos recursos para investimento em máquinas e equipamentos pelo BNDES não está diretamente ligada à classificação do destino do recurso à inovação.

Segundo a ANPEI (2017) para operações que representam apenas aquisição de máquinas/equipamentos, ou modernização de ambientes produtivos, tais situações não são beneficiadas pela Lei do Bem.

Existem diversas linhas de créditos disponíveis no mercado com modalidades que não são diretamente voltadas para projetos de inovação, mas podem ser aplicadas para tal. Como exemplos, estão os créditos direcionados para aquisição de máquinas, micro crédito, créditos para pequenas e micros empresas, e até mesmo os créditos automáticos e os cartões do BNDES. 
Quanto ao número de empresas que captaram os recursos disponibilizados pelo BNDES classificados como inovação, observa-se um número extremamente reduzido quando comparado ao total de usuários dos recursos disponibilizados para o mesmo período.

Este resultado pode ser melhor compreendido quando observado os fatores que dificultam a captação desses recursos apontados por pesquisa desenvolvida pela Federação das Indústrias do Estado do Paraná (FIEP), que destaca algumas das maiores dificuldades apontadas pelas próprias empresas pesquisadas. Dentre esses fatores tem-se destacado o excesso de burocracia, as condições financeiras ofertadas como prazos, formas de pagamentos e juros excessivos. O desconhecimento de editais de fomentos e de linhas de créditos tem se posicionado na sequência, como pode ser observado no Gráfico 3.

Gráfico 3: Dificuldades na captação de recursos para inovação.

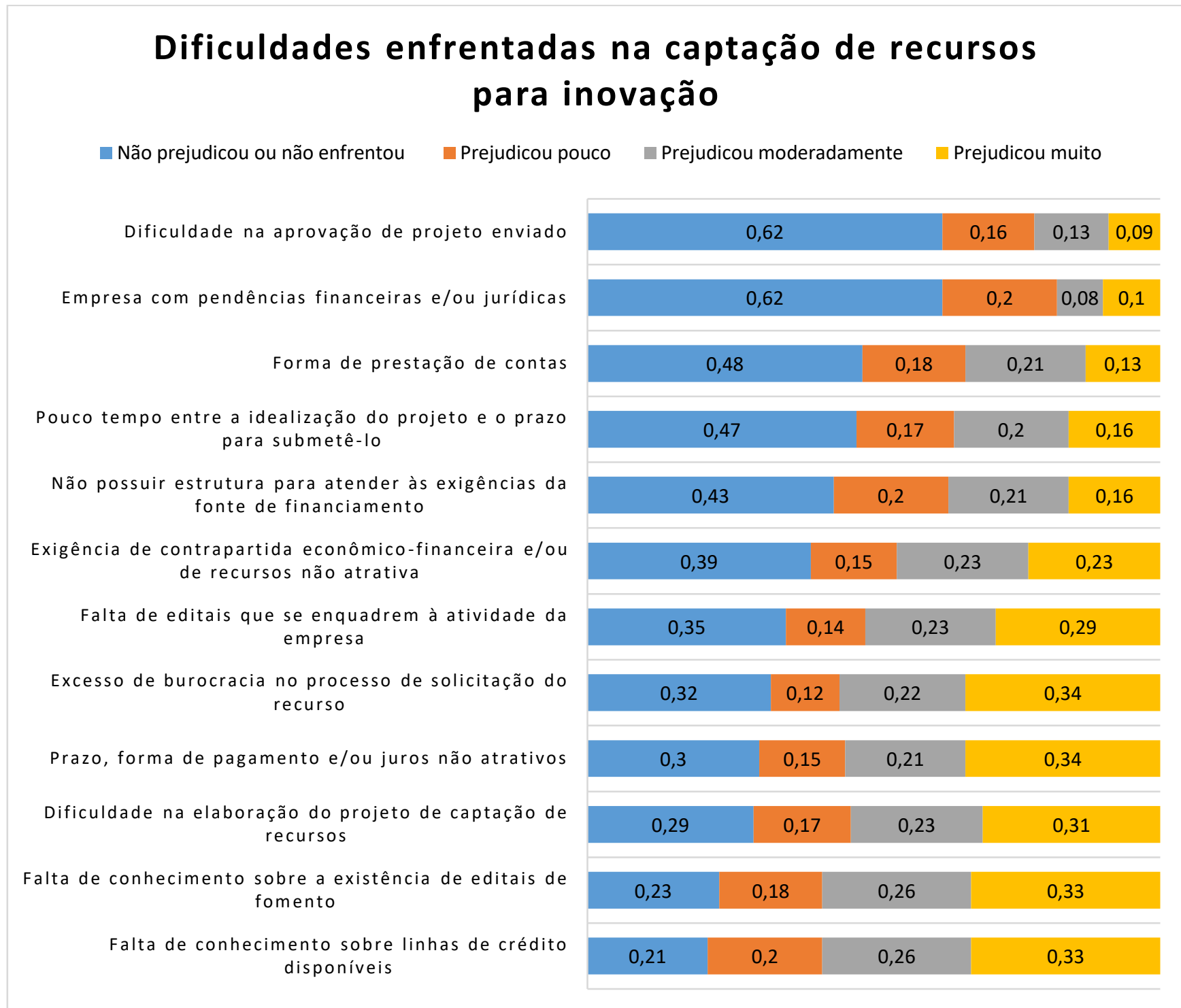

Fonte: Elaborado pelo autor a partir de FIEP (2019).

Na pesquisa PINTEC 2017 também são destacados quatro principais fatores de obstáculos à inovação segundo as empresas: a) riscos econômicos excessivos; b) elevados custos da inovação; c) falta de mão de obra qualificada e d) escassez de fontes de financiamento, todos relacionados a custos e riscos. (IBGE, 2020a). 
Muitas empresas não dispõem de setores distintos para desenvolver atividades de engenharia e PD\&I, tornando difícil a segregação entre essas atividades, dificultando a seleção dos projetos que podem ser incentivados pela Lei do Bem e à avaliação conceitual que o MCTIC faz destes projetos (ANPEI, 2017).

\section{Conclusão}

No contexto quanto aos dados apontados pelo BNDES referentes aos recursos alocados como inovação, comparados aos números de empresas inovadoras informadas pelo IBGE, mesmo quando considerados a inclusão dos números referentes à captação de recursos destinados a aquisição de máquinas e equipamentos por aquele, a resultante ainda evidencia um contraste numérico.

Essa diferença nas quantidades apontadas pode corroborar com os indicadores do próprio IBGE quanto da FIEP, que apontam um alto índice de inovadores que utilizaram recursos próprios tanto para desenvolvimento de seus projetos de inovação quanto para $\mathrm{P} \& \mathrm{D}$.

Em relação ao baixo índice de empresas que inovaram com incentivos do governo, pode ser justificado pelas dificuldades das empresas em captar tais recursos. Nesse quesito ainda pode-se destacar a complexidade processual na captação de recursos externos, classificados com capital de risco. Os óbices para utilização dos incentivos fiscais também são apontados como justificativa para baixa aderência ao incentivo fiscal para inovação, visto que a maioria das empresas são optantes pelo regime de lucro presumido.

Das limitações observadas para o desenvolvimento deste estudo estão a ausência de informações no MCTIC sobre dados de utilização de recursos para empresas inovadoras, assim como dados das empresas beneficiadas pelos incentivos fiscais.

É indiscutível os esforços dispostos pelo Estado para com os incentivos ao desenvolvimento da inovação em nosso país, esforços esses que, apesar de recentes em nossa história, buscam viabilizar o crescimento do país, apoiando seus atores da produção econômica, buscando remeter o país para uma posição de destaque no ranking global de inovação.

Muitos são os programas de incentivo à inovação junto aos diversos agentes de fomento, fundos de apoio, serviços de apoio às micros, pequenas e médias empresas, enfim, recursos públicos de incentivo dispostos pelo Estado, porém, existe um grande vale para ser compensado, na curva que reflete a utilização dos recursos para inovação. O Estado tem desenvolvido um papel de coadjuvante nesse cenário, quando se refere aos desembolsos destinados ao desenvolvimento da inovação tecnológica em nosso país.

Para trabalhos futuros sugere-se estudos com objetivo de avaliar e mensurar os métodos aplicados nas avaliações dos projetos de inovação para liberação de recursos bem como a divulgação de tais recursos junto a pequenas e micros empresas.

\section{Referências}

ANPEI. Associação nacional de pesquisa e desenvolvimento das empresas inovadoras. (2017). Guia da Lei do Bem O que é inovação para a Lei do Bem? http://materiais.anpei.org.br/guialeidobem Versão 1.1 / Dezembro de 2017

BNDES. (2020). Central de Downloads, Dados Sobre Operações De https://www.bndes.gov.br/wps/portal/site/home/transparencia/centraldedownloads/!ut/p/z1/pZJBc9owEIX_Si4Ci2RDXZvJiGQGJpOMgTQJSPbAquDJUdSc NJf342bQ0MKnU59sldrvfeXcrpinIt9morvDJa7PB7zQePsyQbT8NbmMH9iEF6ObyOZpMEwhtG110DHH1SoPz3_yd30Ic0yq7Sh8uMxYsBfaCc8kL7xld0net SukelnVfuegcBFCZWgbgrdCuEVbqQokACqmxsjsr5VlpWr0zonRvNzWFKuk6kZJJgCEBEQMJo01C4iJPCBvCJo4GuczL8t35iWj8dLCbv0VDdud2fjHfoi3 hK6L0xtCVdB7hYsJCOGIaaUUXVDnisCprQToKaI9_FAD27QrS6ay_COcjOM_Cw4bbazbGhotZFI6z_iRh7w0nMqyRwfAog1GfLvdKtnShja1xG7_EfH06 XPIJGS-v70xFNcA6O9fPF09Z97gJrbncl_rW-qcxbjCKzcSCtt79liufKcV8CCKBt214n19uafS3WGlcAI2x_k3UKSPSH8YJF4g60bqyhwcnJjwH8xVxmH8Q00qRd1zF4Jzwd30x-jr2SyxPfX9ieMrAGg/dz/d5/L2dBISEvZ0FBIS9nQSEh/\#

BNDES. (2020). Consulta a operações do BNDES 2020. https://www.bndes.gov.br/wps/portal/site/home/transparencia/consulta-operacoes-bndes/

Calmanovici, C. E. (2011). A inovação, a competitividade e a projeção mundial das empresas brasileiras. Revista Usp, (89), 190-203.

Salerno, M. S., \& Daher, T. (2006). Política industrial, tecnológica e de comércio exterior do governo federal (PITCE): balanço e perspectivas. Brasília: Agência Brasileira de Desenvolvimento Industrial. 
Research, Society and Development, v. 11, n. 3, e22411326399, 2022

(CC BY 4.0) | ISSN 2525-3409 | DOI: http://dx.doi.org/10.33448/rsd-v11i3.26399

FIEP. Federação da Indústria do Estado do Paraná. (2019) Bússola Da Inovação: Perfil De Inovação Industrial, Paraná 2019, Curitiba, Senai-Pr. http://bussolasdaindustria.org.br/inovacao/\#

Gil, A. C. (2002). Como elaborar projetos de pesquisa (4, 175). Atlas.

Guimarães, F. C. D. M. S. (2010). A política de incentivo à inovação: inovação, desenvolvimento econômico e política tecnológica. Parcerias Estratégicas, 5(9), 121-128.

IBGE. (2016). Pesquisa de Inovação 2014. Coordenação de Indústria. https://biblioteca.ibge.gov.br/visualizacao/livros/liv99007.pdf

IBGE (2020a). Pintec 2017, livro informativo. https://biblioteca.ibge.gov.br/visualizacao/livros/liv101706_informativo.pdf.

IBGE (2020b). Pesquisa de Inovação - PINTEC, Principais resultados 2014. https://www.ibge.gov.br/estatisticas/multidominio/ciencia-tecnologia-einovacao/9141-pesquisa-de-inovacao.html?edicao=9142\&t=destaques .

IBGE (2020c). Pesquisa de Inovação - PINTEC, Principais resultados 2017. https://www.ibge.gov.br/estatisticas/multidominio/ciencia-tecnologia-einovacao/9141-pesquisa-de-inovacao.html?edicao $=27431 \& \mathrm{t}=$ destaques

IPEA - Instituto de Pesquisa Econômica Aplicada (2008). Política de Incentivo à Inovação Tecnológica no Brasil. Brasília.

Kannebley Jr, S., \& Porto, G. (2012). Incentivos Fiscais à Pesquisa, Desenvolvimento e Inovação no Brasil. Uma avaliação das Políticas Recentes. Banco Interamericano de Desenvolvimento (BID).

Lall, S. (1992). Technological capabilities and industrialization. World Development, 20(2), 165-186.

De Oslo, O. M. (2005). Diretrizes para coleta e interpretação de dados sobre inovação. Organização para a Cooperação e Desenvolvimento Econômico.

Matias-Pereira, J. (2015). Uma avaliação das políticas públicas de incentivo a inovação tecnológica no Brasil: a Lei do Bem. Parcerias Estratégicas, 18(36), 221-250.

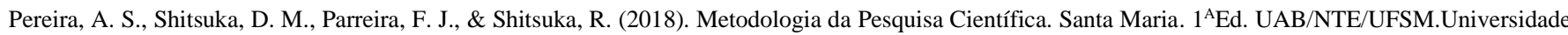
Federal de Santa Maria. Santa Maria RS

Porto, G. S., \& Memória, C. V. (2019). Incentivos para inovação tecnológica: um estudo da política pública de renúncia fiscal no Brasil. Revista de Administração Pública, 53, 520-541.

Rauen, C. V. (2016). O novo marco legal da inovação no Brasil: o que muda na relação ICT-Empresa? Radar, 43, 21-35.

Schumpeter, J. A. (1982). Teoria do desenvolvimento econômico: uma investigação sobre lucros, capital, crédito, juros e o ciclo econômico. Col. Os Economistas. Abril Cultural.

Ribeiro, M. T. F. R. (2006). Paulo Bastos Tigre-Gestão da Inovação: a economia da tecnologia no Brasil. Revista Brasileira de Inovação, 5(2), $479-785$.

Scaff, L. C. M., \& Pereira L. F. F (2021). Institucionalismo \& Inovação: A Lei do bem como instituição extrativista no ecossistema inovativo nacional. Revista Estudos Institucionais. 7(2), 629-648. doi:10.21783/rei.v7i2.582

Veloso Filho, F. D. A., \& Nogueira, J. M. (2006). Sistemas de inovação e promoção tecnológica regional e local no Brasil. Interações (Campo Grande), 8, 107117.

Vicente, R. H., \& Lopes, P. D. L. (2015, October). A importância de investimentos em inovação tecnológica como fator chave para o desenvolvimento econômico. In XII Simposio de excelência em gestão e tecnologia-SEGeT. 